on account of their acceleration in the electric fields of the electromagnetic radiation through which they move, whereas synchrotron radiation is caused by the acceleration associated with spiralling round quasi-static magnetic field lines. Large magnetic fields, considerably in excess of 100 gauss, are needed to ensure that the synchrotron process is dominant, and such fields are not easy to come by with some models of the 'explosive event'. What Colvin has done is to suggest a twostage process for generating the radiation from QSOs that is effective even with magnetic fields as small as 30 gauss.

The basic fact made use of in the theory is that for some radiation frequencies a plasma containing a magnetic field has a refractive index greater than 1. Highly relativistic particles particles therefore move through it at a speed exceeding the speed of light in the medium. In consequence they produce Čerenkov radiation-the electromagnetic equivalent of the shock wave generated in air by supersonic aircraft. Colvin estimates that under QSO conditions the Cerenkov photons could have a characteristic frequency of $10^{8} \mathrm{~Hz}$. The number of photons could be sufficient for them to make the relativistic electrons of lower energy then undergo inverse Compton energy loss and produce visible radiation of the intensity that is observed.

\section{Strength functions in rare earths}

\section{from P. E. Hodgson}

ACCORDING to the simple single-particle model of nuclei each nucleon occupies a state with definite energy and quantum numbers, and these are the eigenstates of a one-body potential. Such states are selectively populated by one-nucleon transfer reactions, and a study of these reactions enables the energies of the single-particle states to be determined.

Thus, for example, a $(d, p)$ reaction simply puts a neutron into one of the unoccupied single-particle states of the residual nucleus, so that the energy spectrum of the emitted protons reflects the energies of the unoccupied states in the residual nucleus.

In practice such a simple result is only found even approximately for the low-lying states in reactions depositing a nucleon into a closed shell nucleus. In other cases the single-particle strength is distributed over a range of excitation energies. This takes place in two different ways; either the singleparticle strength can be 'spread' over a large number of states in the residual nucleus according to a Lorentzian distribution or it can be 'fragmented' by

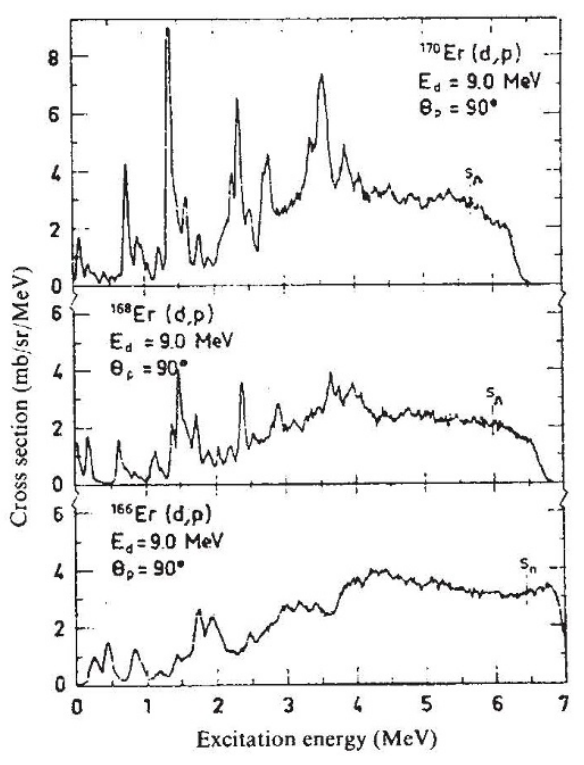

Fig. 1 Energy spectrum for the (d,p) reaction at $9 \mathrm{MeV}$ on three isotopes of erbium.

residual interactions that are not taken into account by the one-body potential. The latter can result from a coupling of the single-particle states to vibrational degrees of freedom or from coupling between the one and three quasiparticle states. The spreading width is expected to increase with excitation energy while the fragmentation width remains almost constant.

It is important to try to find out the relative importance of spreading and fragmentation, for only if the fragmentation is less than the spreading is it possible to identify and assign quantum numbers to the higher states.

This problem has recently been studied in the rare earth region by Back, Bang, Bjørnholm, Hattula, Kleinheinz and Lien (Nucl. Phys., A222, 377 ; 1974). They irradiated several isotopes of the rare earth nuclei gadolinium, dysprosium, erbium and ytterbium with $9 \mathrm{MeV}$ deuterons and measured the energy distribution of the outgoing protons. Plotted on an appropriate energy scale this gives immediately a measure of the distribution of the neutron single-particle strength, or neutron strength function as it is called. A typical result, that for erbium, is shown in Fig. 1; the peaks in the spectra move towards lower energies for the heavier nuclei, which is what would be expected from the Nilsson model, and that there is structure below about $4 \mathrm{MeV}$ but no structure at higher energies.

The theoretical cross sections for these reactions were calculated using the distorted wave theory of stripping reactions to single particle states in deformed nuclei. The wavefunctions were calculated as the eigenvalues of a deformed Saxon-Woods potential. To enable the results of this calculation to be compared with the data the peaks were broadened by multiplying by a Lorentz factor. The results for erbium are shown in Fig. $2 a$ and show qualitative agreement. The cumulative cross section (Fig. 2b) is in good accord with the data, indicating that the total neutron strength is preserved and that higher order processes are relatively unimportant. It is, however, difficult to make unique quantum number assignments to the peaks at higher energies.

This work gives a remarkably clear picture of the behaviour of neutron single-particle strengths as a function of excitation energy, and shows that both spreading and fragmentation take place.
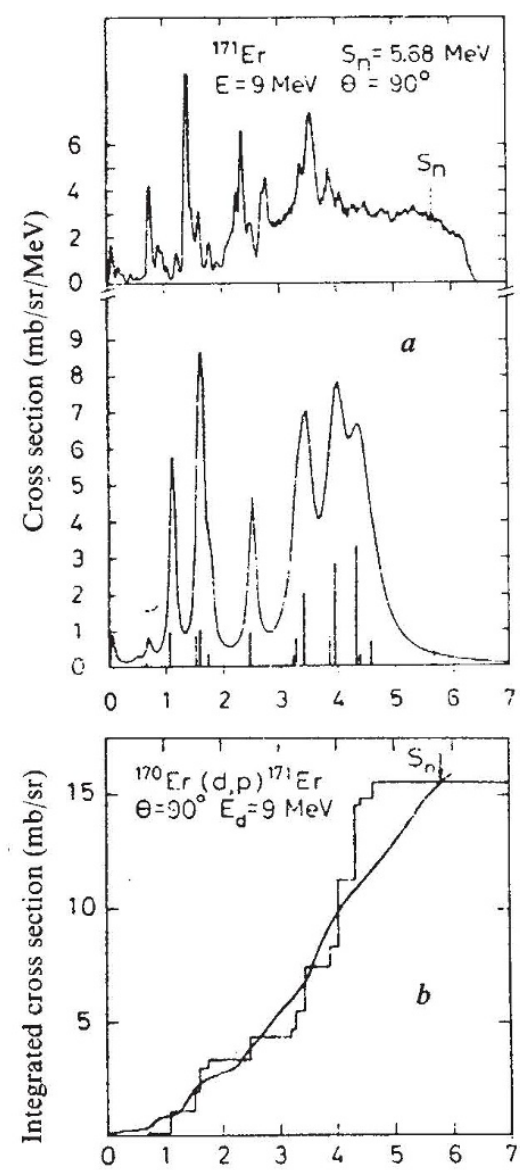

Excitation energy (MeV)

Fig. $2 a$, Experimental (upper) and theoretical (lower) differential cross sections calculated in a deformed Saxon-Woods potential. The vertical lines represent the total stripping strength of the rotational band corresponding to the deformed singleparticle state. The smooth curve represents this strength distributed according to the Lorentz faotor. $b$, Comparison of the cumulative integrated experimental cross section and the theoretical cross section for energies below the neutron separation energy $S_{\mathrm{n}}$. 